\title{
Uterine large cell neuroendocrine carcinoma with unusual colonic metastasis
}

\author{
Antonio leni', Giuseppe Angelico', Rosalba De Sarro' ${ }^{2}$, Francesco Fleres ${ }^{3}$, Antonio Macri ${ }^{3}$, Giovanni Tuccari ${ }^{1}$ \\ 'Department of Human Pathology of Adult and Evolutive Age "Gaetano Barresi”, Section of Anatomic Pathology, University of Messina, 98125 Messina, Italy. \\ ${ }^{2}$ Student in Medicine and Surgery, University of Messina, 98125 Messina, Italy. \\ ${ }^{3}$ Department of Human Pathology of Adult and Evolutive Age "Gaetano Barresi", Section of General Surgery, University of Messina, Azienda Ospedaliera \\ Universitaria "Policlinico Gaetano Martino”, 98125 Messina, Italy.
}

Correspondence to: Prof. Giovanni Tuccari, Department of Human Pathology of Adult and Evolutive Age "Gaetano Barresi”, Section of Anatomic Pathology, University of Messina, 98125 Messina, Italy. E-mail: tuccari@unime.it

How to cite this article: Ieni A, Angelico G, De Sarro R, Fleres F, Macrì A, Tuccari G. Uterine large cell neuroendocrine carcinoma with unusual colonic metastasis. J Cancer Metastasis Treat 2017;3:144-9.

\author{
Article history: \\ Received: 27-02-2017 \\ Accepted: 23-05-2017 \\ Published: 16-08-2017 \\ Key words: \\ Neuroendocrine carcinoma, \\ metastasis, \\ uterus, \\ differential diagnosis, \\ immunohistochemistry
}

\begin{abstract}
A 78-year-old female patient arrived at our practice complaining of progressive abdominal increase and presenting a clinical picture of intestinal obstruction. At physical examination, the abdomen appeared distended, moderately painful with the presence of a mass of hard consistency. Abdominal computed tomography scan showed a large hypodense pelvic mass that indicated a compression and lateral deviation of the uterus and bladder. Microscopically, the mass showed a uniform solid pattern, composed of medium and large-sized cells with hyperchromatic and pleomorphic nuclei demonstrating high mitotic activity and diffuse immunoreactivity for estrogen receptors and synaptophysin. A diagnosis of uterine poorly differentiated large cell neuroendocrine carcinoma, arising in the endometrium with an unusual colonic metastatic localization, was made.
\end{abstract}

\section{INTRODUCTION}

Uterine neuroendocrine carcinomas are rare and highly malignant tumors, morphologically subdivided into small and large cell according to their nuclear size, presenting as pure or combined forms, either associated with endometrioid adenocarcinoma or as a component of a malignant mixed müllerian tumor. ${ }^{[1,2]}$ It has been reported that the primary site of neuroendocrine tumors (NETs) is unknown in about $13 \%$ of patients, ${ }^{[3]}$ although the exact incidence of unknown primary NETs has not been fully determined. ${ }^{[4]}$ To identify the nature as well as the primary site of NETs, the immunohistochemical approach appears to be the most useful approach. It allows a correct characterization identifying site-specific transcription factors, such as thyroid transcription factor 1 (TTF-1) and CDX2. ${ }^{[5]}$ In particular, nuclear TTF-1 staining is effective in more than $50 \%$ of pulmonary carcinoids but only rarely in gastrointestinal NETs, while nuclear CDX2-staining is revealed in gastrointestinal NETs but seldom in pulmonary carcinoids. ${ }^{[5]}$ Moreover, in

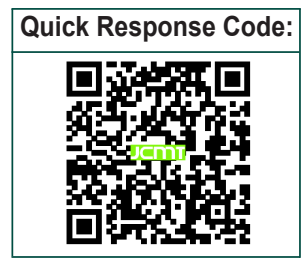


poorly differentiated neuroendocrine carcinomas, transcription factors are expressed irrespectively of primary neoplastic site, causing diagnostic problems..$^{[5]}$

We report a case of a large cell neuroendocrine carcinoma (LCNEC), initially developed in uterus and clinically silent, with an unusual colonic metastatic localization during the progression of the disease.

\section{CASE REPORT}

A 78-year-old female patient arrived in our practice complaining of progressive abdominal increase and presenting a clinical picture of intestinal obstruction. Her past medical history was characterized by an episode of intestinal perforation six months before in another hospital including right hemicolectomy. The histopathological examination of the surgical specimen suggested a poorly differentiated adenocarcinoma with serosal invasion (T4) and lymph node metastases (N1). The patient received 5 successive cycles of chemotherapy and underwent a follow-up examination. Six months later she was admitted to our hospital; at physical examination, the abdomen appeared distended, moderately painful with the presence of a mass of hard consistency. Tumor markers were normal. The patient underwent an abdominal computed tomography scan showing a large hypodense pelvic mass of $21 \mathrm{~cm} \times 15 \mathrm{~cm} \times 19 \mathrm{~cm}$, with peritoneal implants, which caused an important distortion and lateral deviation of the uterus and bladder. Regional lymph nodes were enlarged. The patient underwent debulking surgery. A large neoplastic mass occupied the abdomen from the transverse colon to the pelvis, infiltrating the small intestine, the anterior parietal and prevesical peritoneum, the sigmoid colon and proximal rectum. The small intestine was disease free for about $160 \mathrm{~cm}$, being dislocated in the left upper quadrant. Surgeons isolated the lesion from the retroperitoneal structures and then removed en bloc the tumor, the small intestine, a small portion of the residual transverse colon, the sigmoid colon, the proximal rectum, the uterus, the uterine annexes, pelvic and anterior parietal peritoneum [Figure 1]. Finally, reconstruction of the digestive tract was performed by creating a mechanical termino-lateral ileo-transverse anastomosis and terminal colostomy. Postoperatively the patient was admitted to the intensive care unit for 7 days. Gastrografin enema, performed on the 8th post-operative day to control the ileo-colic anastomosis, demonstrated the absence of any leakage. Three months later the patient was alive, but was subsequently lost to follow-up.

Representative surgical specimens taken from uterus, colon, peritoneum and small intestine were fixed in $10 \%$ buffered formaldehyde for $24 \mathrm{~h}$, completely sampled, routinely processed and paraffin-embedded at $56{ }^{\circ} \mathrm{C}$. Four micron thick sections were cut and routinely stained with haematoxylin and eosin. Immunohistochemical stainings were performed with DAKO Link 48 automated system (DakoCytomation, Copenhagen, Denmark) using commercially obtained mono-or polyclonal antibodies [Table 1].

Microscopically the mass appeared to have developed

Table 1: Source, working dilution and immunostainings regarding the panel of utilized antisera

\begin{tabular}{|c|c|c|c|}
\hline Antibody & Clone and dilution & Company & Staining \\
\hline SMA & 1A4, w.d. 1:100 & DakoCytomation, Copenhagen, Denmark & - \\
\hline Calretinin & DAK Calret 1, w.d. 1:50 & DakoCytomation, Copenhagen, Denmark & - \\
\hline CD10 & 56C 6, w.d. $1: 80$ & DakoCytomation, Copenhagen, Denmark & - \\
\hline CD56 & $123 \mathrm{C} 3$, w.d. $1: 50$ & DakoCytomation, Copenhagen, Denmark & $-/+$ \\
\hline CD117 & C-kit, 1:400 & DakoCytomation, Copenhagen, Denmark & - \\
\hline CK & AE1/AE3, w.d. 1:50 & DakoCytomation, Copenhagen, Denmark & - \\
\hline CK 7 & OV-TL $12 / 30$, w.d. $1: 50$ & DakoCytomation, Copenhagen, Denmark & - \\
\hline CK 20 & Ks 20.8, w.d. 1:50 & DakoCytomation, Copenhagen, Denmark & - \\
\hline CDX2 & DAK-CDX2, w.d. 1:50 & DakoCytomation, Copenhagen, Denmark & - \\
\hline Chromogranin A & DAK-A3, w.d. 1:200 & DakoCytomation, Copenhagen, Denmark & ++ \\
\hline Desmin & D33, w.d. 1:100 & DakoCytomation, Copenhagen, Denmark & - \\
\hline EMA & E29, w.d. 1:100 & DakoCytomation, Copenhagen, Denmark & $-/+$ \\
\hline ER & 1D5, w.d. 1:50 & DakoCytomation, Copenhagen, Denmark & +++ \\
\hline Ki67 & MIB-1, w.d. 1:75 & DakoCytomation, Copenhagen, Denmark & $80 \%$ \\
\hline MLH1 & G168-728, w.d. 1:100 & Cell Marque, Rocklin, California, USA & ++ \\
\hline $\mathrm{MSH} 2$ & G219-1129, w.d. 1:100 & Cell Marque, Rocklin, California, USA & ++ \\
\hline MSH6 & SP93, w.d. 1:50 & Cell Marque, Rocklin, California, USA & ++ \\
\hline PAX-8 & EP298, w.d. 1:500 & Cell Marque, Rocklin, California, USA & - \\
\hline $\mathrm{PgR}$ & PgR 636, w.d. 1:50 & DakoCytomation, Copenhagen, Denmark & $-/+$ \\
\hline S100 & Polyclonal, w.d. 1:50 & DakoCytomation, Copenhagen, Denmark & - \\
\hline Synaptophysin & DAK-SYNAP, w.d. 1:50 & DakoCytomation, Copenhagen, Denmark & ++ \\
\hline Vimentin & V9, w.d. $1: 50$ & DakoCytomation, Copenhagen, Denmark & - \\
\hline
\end{tabular}

CK: cytokeratin; EMA: epithelial membrane antigen; ER: estrogen receptor; PgR: progesteron receptor; CD56: neural cell adhesion molecules; CD117: tyrosine chinase receptor; CDX2: caudal-related homeobox transcription factor; MLH1: MutL homolog 1 colon cancer non polypois type 2; MSH2: human homolog of the Escherichia Coli mismatch repair gene mutS; MSH6: protein similar to the MutS protein; PAX-8: protein member of the paired box family of transcription factors; SMA: smooth muscle actin; S100: protein S-100; w.d.: work dilution 
in the uterine corpus involving the myometrium and serosal layer, with an infiltration of the colonic wall indicating lymph node metastases as well. The proliferation showed a uniform solid pattern, with complete absence of glandular differentiation and areas of geographic necrosis [Figure 2A]; it was characterized by medium and large-sized cells with hyperchromatic and pleomorphic nuclei, prominent nucleoli and high mitotic activity [Figure 2B]. Immunohistochemistry revealed a diffuse positivity for estrogen receptor (ER) [Figure 3A], chromogranin-A [Figure 3B], synaptophysin [Figure 3C], MLH1, MSH2, MSH6 and a partial staining for EMA, CD56 and progesterone receptor $(\mathrm{PgR})$. No immunostainings
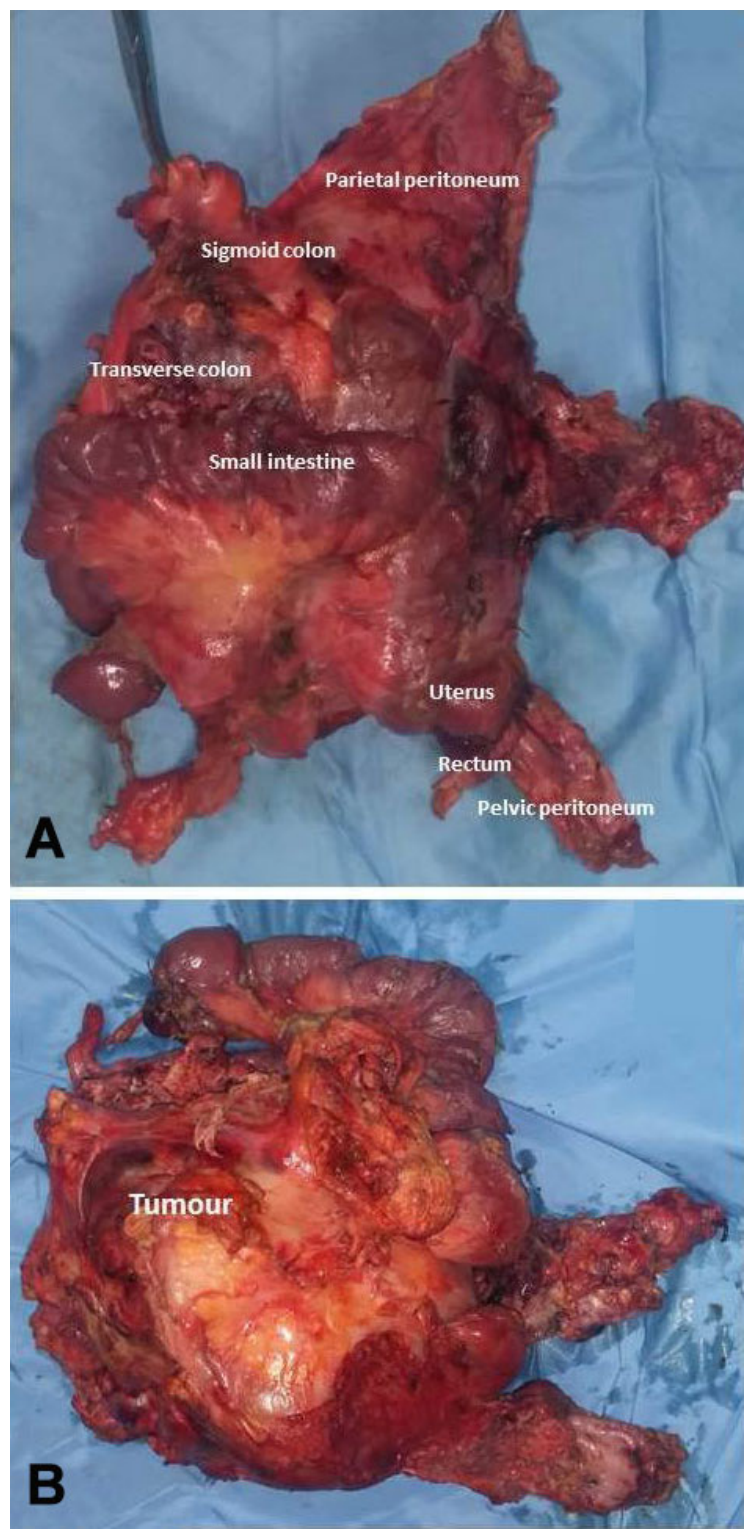

Figure 1: Grossly anterior and posterior appearance of the surgical specimen. (A) Macroscopically, in the anterior view, the relationships between neoplasia and adjacent anatomical structures are seen; (B) grossly, the tumor mass is easily appreciable at the posterior view of the surgical sample were present for CK, CK 7, CK20, CDX2, TTF1, Pax8, CD10, vimentin, desmin and CD99. The growth fraction, assessed with Ki67, revealed a positivity of more than $80 \%$ of neoplastic elements. A diagnosis of infiltrating poorly differentiated LCNEC was made, based on synaptophysin, chromogranin-A, ER and $\mathrm{PgR}$ immunoreactivity. The diagnosis was classified as a primary tumor of the uterus, with extensive colonic and peritoneal spread. In light of these findings, we took the opportunity to re-examine the original neoplastic paraffin-block taken at the colonic level during the first surgical procedure. Histologically the colonic wall was extensively ab-extrinseco infiltrated by a highly cellular solid proliferation [Figure 4A], suggestive of a poorly differentiated adenocarcinoma, but absolutely unreactive for CK20 [Figure 4B], a marker usually positive in colonic cancer. Finally, a heterogeneous, well evident, cytoplasmic staining for chromogranin-A (Figure 4B, inset) was appreciable in neoplastic elements. These morphological data were consistent with a diagnosis of colonic parietal infiltration by aggressive neuroendocrine carcinoma.

\section{DISCUSSION}

NETs are more generally identified in the gastrointestinal tract, pancreas, lung and thymus, while in the female reproductive tract they account for about $2 \%$ of all gynecologic cancer ${ }^{[6,7]}$ According to World Health Organization classification, NETs are classified in two principal groups: poorly differentiated neuroendocrine carcinomas (NECs) and welldifferentiated NETs. ${ }^{[8]}$ NECs include small and large cell neuroendocrine carcinoma, while NETs include typical and atypical carcinoids..$^{[8]}$

Poorly differentiated LCNEC of the endometrium is a very uncommon tumor representing only $0.8 \%$ of endometrial cancers and they are considered particularly aggressive neoplasms with a tendency for early metastases and poor outcomes. ${ }^{[9]}$ Usually, endometrial NECs are combined with other epithelial neoplasms; in detail, $50-80 \%$ of cases are admixed with FIGO grade 1 or 2 endometrioid adenocarcinoma. ${ }^{[6,7]}$ To explain this intriguing association it has been hypothesized that some endometrial NECs may arise from the neuroendocrine component of endometrioid carcinomas. ${ }^{[10]}$ Although the possibility that an abdominal NEC may secondarily develop due to chemotherapy for an original endometrial carcinoma should be mentioned, nevertheless in the present case this option should be excluded since the first diagnosis and consequently the therapeutical approach were based on colonic poorly differentiated carcinoma. It has also been suggested that these tumors can be 
derived from pluripotent stem cells with the possibility for both neuroendocrine and glandular endometrioid differentiation. ${ }^{[11]}$

In the current literature, 15 cases of endometrial LCNEC have been described in patients with a mean age of 64 years, 8 of which cases are pure and 7 are associated with another component. ${ }^{[8]}$ In particular, the pure form LCNEC is characterized by solid sheets with organoid, trabecular or cordlike patterns including peripheral palisading and necrosis areas. ${ }^{\left[{ }^{[3]}\right.}$ The neoplastic cells have large and abundant eosinophilic cytoplasm with vesicular high-grade nuclei, prominent nucleoli and frequent mitotic figures. ${ }^{[9]}$ The confirmation of neuroendocrine differentiation is based on neuroendocrine markers, such as chromogranin, synaptophysin and CD56. ${ }^{[5]}$ In this case, the diagnosis of endometrial LCNEC was based on neuroendocrine appearance, particularly the neuroendocrine marker expression (synaptophysin and partial CD56 reactivity). In differential diagnoses, endometrial NECs should be distinguished from other tumors characterized by nuclear high-grade features with a predominantly solid growth pattern, such as carcinosarcoma, undifferentiated endometrial sarcoma, solid pattern of serous carcinoma and undifferentiated endometrial carcinoma (UEC). However, the most problematic differential diagnosis is represented by UEC, in which a focal neuroendocrine differentiation $(<10 \%)$, with 1 or more neuroendocrine markers, has been demonstrated in $41 \%$ in UEC series; ${ }^{[12]}$ therefore, the expression of neuroendocrine markers in more
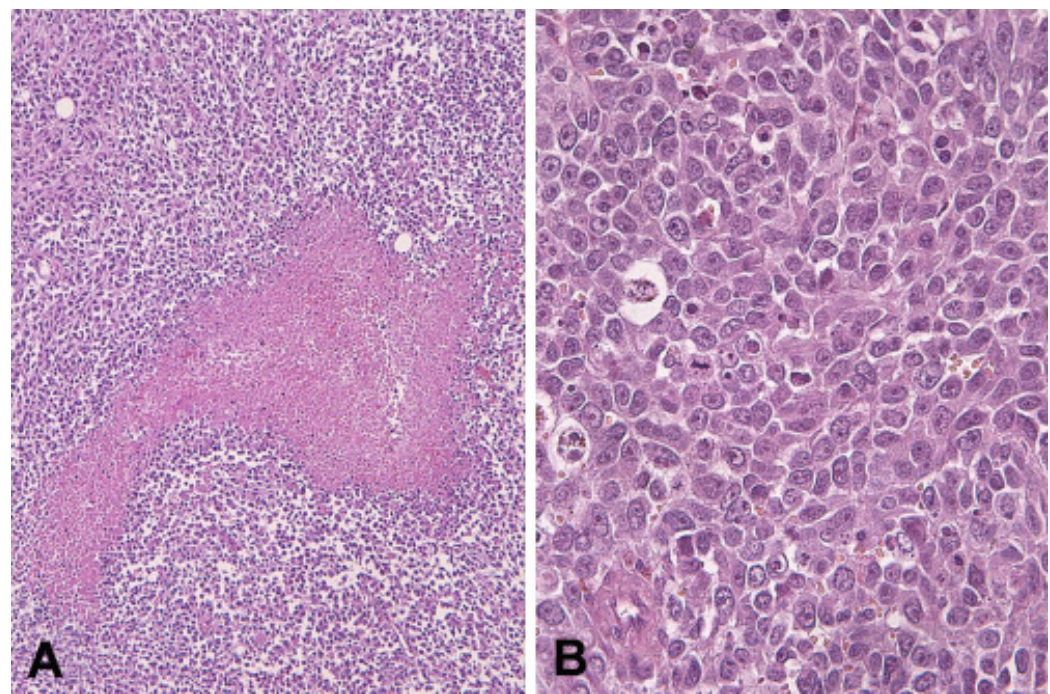

Figure 2: Microscopically the malignant proliferation presented a solid pattern with areas of necrosis (A, hematoxylin-eosin stain, $\times 200)$, composed by medium or large-sized cells with hyperchromatic and pleomorphic nuclei, prominent nucleoli and high mitotic activity (B, hematoxylin-eosin stain, $\times 400$ )

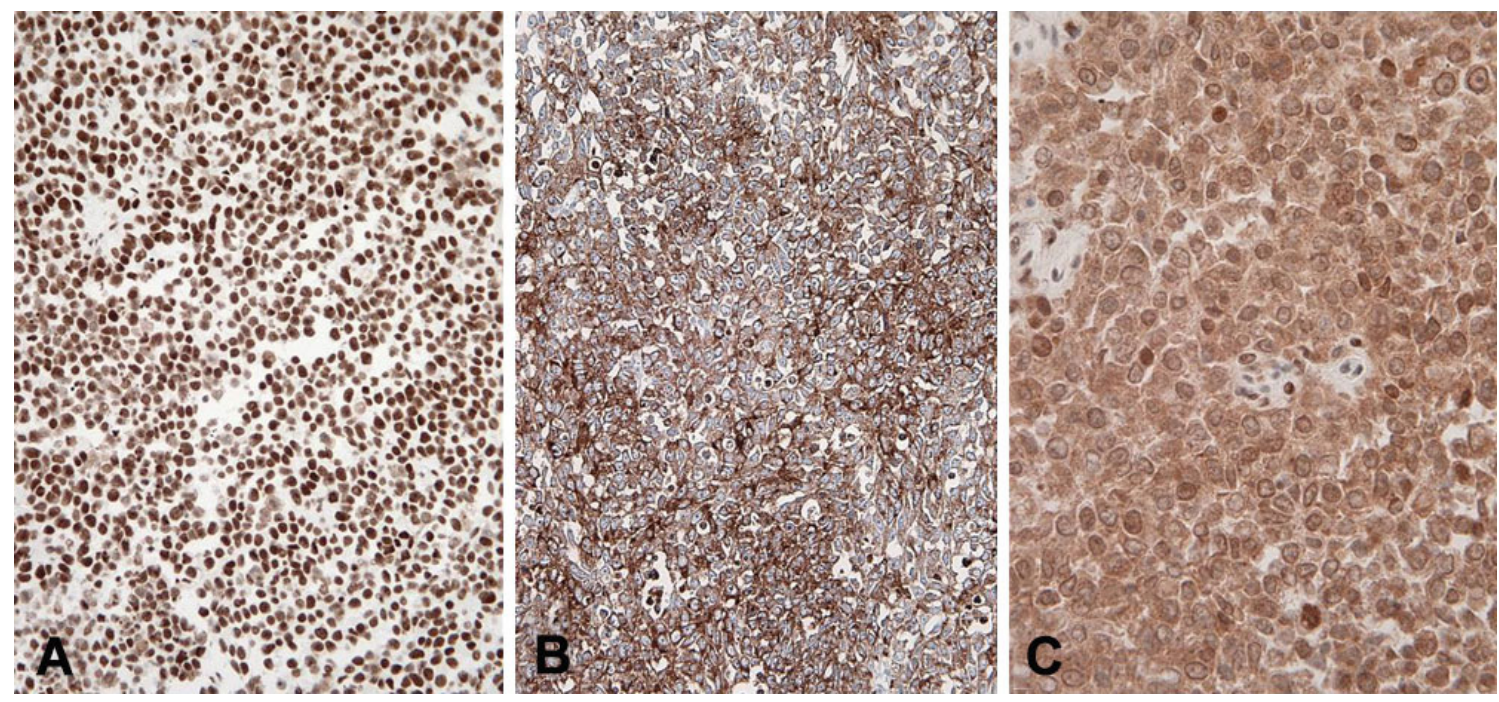

Figure 3: Immunohistochemistry revealed diffuse nuclear reactivity for estrogen receptor $(A, \times 400)$; strong and uniform cytoplasmic staining for chromogranin-A $(B, \times 400$, haematoxylin nuclear counterstain) as well as synaptophysin $(C, \times 400$, haematoxylin nuclear counterstain) 


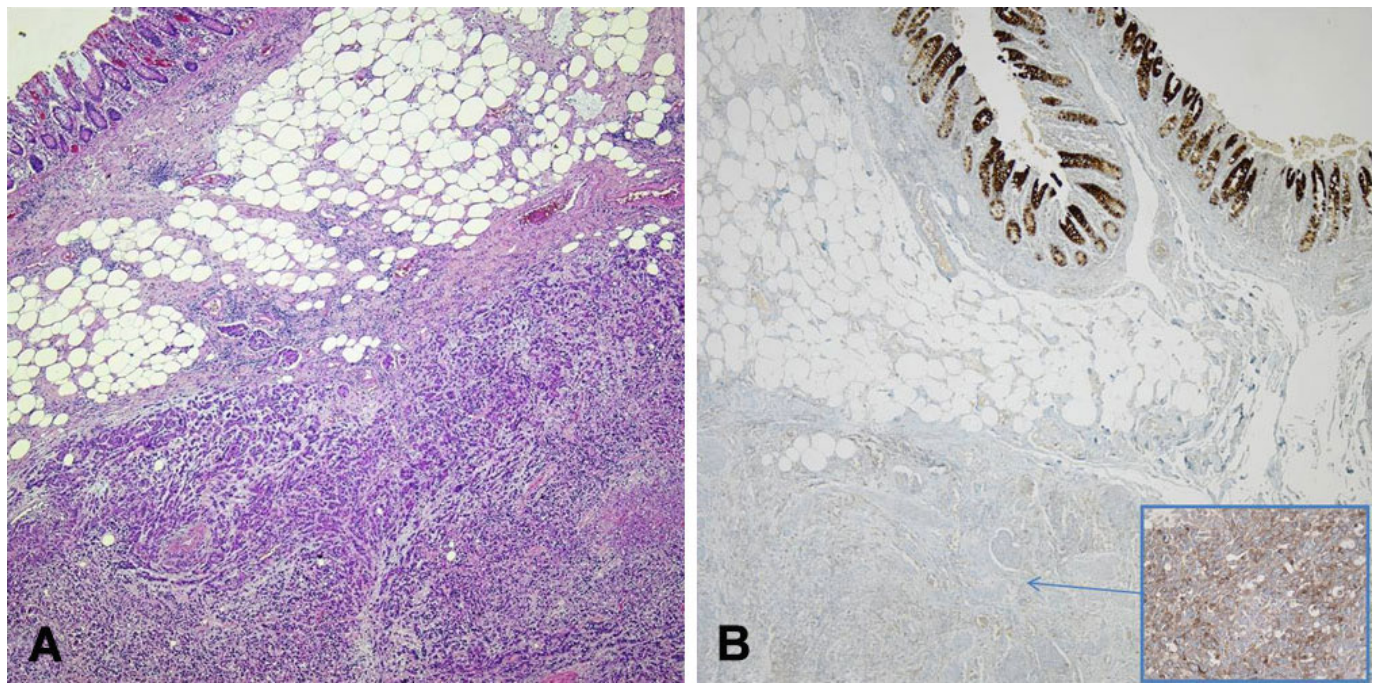

Figure 4: At lower magnification the neoplastic proliferation presented an infiltrative extrinsic pattern of the colonic wall in the first surgical sample (A, $\times 120$, hematoxylin-eosin stain); the neoplasm was unreactive for CK20, (note the positive control of the normal mucosa). The inset showed a patchy not uniform immunoireactivity for chromogranin-A in neoplastic elements $(\times 240$, haematoxylin nuclear counterstain)

than $20 \%$ of tumor cells is required to support the diagnosis of endometrial NECs. ${ }^{[13]}$

In our case, the most intriguing difficulty was to discriminate between a primary uterine tumor and a uterine metastasis from the previous diagnosed colonic NEC. Consequently, we initially performed an immunohistochemical analysis with typical positive markers of the female genital tract, such as ER and $\mathrm{PgR}$, since these receptors are usually considered useful to define the origin of unknown metastatic carcinoma. ${ }^{[14,15]}$ However, the neoplastic proliferation in the present case was strongly positive for ER and negative for CDX2; therefore, it was in contrast to gastrointestinal NETs, characterized by a variable nuclear CDX2 and a negativity for ER and PgR. ${ }^{[5]}$ According to these immunohistochemical findings, we have proposed to apply the same immunohistochemical panel to the colon specimens formerly removed in another hospital, that have confirmed our results supporting the diagnosis of primitive uterine LCNEC.

No prognostic data have been available until now for uterine LCNEC, while only survival data have been reported for cervical small cell neuroendocrine carcinoma (SCNEC). These have showed progression free survival and overall survival (OS) rates of $22 \%$ and $30 \%$, respectively, and a median progression time of 9.1 months. ${ }^{[16]}$ Recently, uterine LCNEC cases have been associated with microsatellite instability (MSI); ${ }^{[17]}$ in detail, by immunohistochemistry it has been showed a mismatch-repair protein immunoexpression in about $44 \%$ of uterine NECs cases, with a prevalence of MLH1/PMS2. ${ }^{[17]}$ However, an intense nuclear positivity with MLH1, MSH2 and MSH6 was observed in our case. Even though it has been demonstrated that a subset of gastrointestinal NECs exhibiting MSI showed a better prognosis than NECs without these features,${ }^{[18]}$ in uterine NECs the presence of MSI does not appear to be associated with a good prognosis. ${ }^{[17]}$

Currently, there is no consensus about the standard treatment of these tumors with either adjuvant chemotherapy or with radiotherapy. ${ }^{[19]}$ In cervical SCNEC cases, it has been suggested that patients who received platinum-based chemotherapy had both a 3-year recurrence-free survival (RFS) and a 3-year OS of $83 \%$, while those not treated with chemotherapy exhibited RFS and OS of $0 \%$ and $20 \%$, respectively. ${ }^{[16]}$ In our case, the surgical procedure was undertaken to debulk the colonic mestastatic localization with additional chemotherapy; nevertheless, taking into consideration the aggressive course and poor prognosis of LCNEC, characterized by the low therapeutic response with a progression of disease, the opportunity of neoadjuvant chemotherapy approach prior to surgery should be considered in the future.

\section{DECLARATIONS}

\section{Authors' contributions}

Participated in the study design and analysis: A. Ieni, G. Angelico, F. Fleres, A. Macrì, G. Tuccari

Contributed to acquisition of literature data: $G$. Angelico, R. De Sarro, F. Fleres

Performed the surgical procedures: A. Macrì, F. Fleres Realized the morphological study: A. leni, G. Angelico, R. De Sarro, G. Tuccari

Drafted the manuscript: A. leni, A. Macrì, G. Tuccari Read and approved the final manuscript: A. leni, G. Angelico, R. De Sarro, F. Fleres, A. Macrì, G. Tuccari 


\section{Financial support and sponsorship}

None.

\section{Conflicts of interest}

There are no conflicts of interest.

\section{Patient consent}

A patient's written consent was achieved for scientific institutional purpose.

\section{Ethics approval}

All procedures were performed in accordance with the Helsinki declaration. No further ethical approval was necessary to perform the diagnostic routine analysis as well as the immunohistochemical algorithm.

\section{REFERENCES}

1. D'Antonio A, Addesso M, Caleo A, Guida M, Zeppa P. Small cell neuroendocrine carcinoma of the endometrium with pulmonary metastasis: a clinicopathologic study of a case and a brief review of the literature. Ann Med Surg (Lond) 2015;5:114-7.

2. Matsumoto H, Nasu K, Kai K, Nishida M, Narahara H, Nishida H. Combined large-cell neuroendocrine carcinoma and endometrioid adenocarcinoma of the endometrium: a case report and survey of related literature. J Obstet Gynaecol Res 2016;42:206-10.

3. Bergsland EK, Nakakura EK. Neuroendocrine tumors of unknown primary: is the primary site really not known? JAMA Surg 2014;149:889-90.

4. Lee HS, Han HS, Lim SN, Jeon HJ, Lee HC, Lee OJ, Yun HY, Lee KH, Kim ST. Poorly differentiated neuroendocrine carcinoma in a perigastric lymph node from an unknown primary site. Cancer Res Treat 2012;44:271-4.

5. Schmitt AM, Blank A, Marinoni I, Komminoth P, Perren A. Histopathology of NET: current concepts and new developments. Best Pract Res Clin Endocrinol Metab 2016;30:33-43.

6. Lopes Dias J, Cunha TM, Veloso Gomes F, Callé C, Félix A. Neuroendocrine tumours of the female genital tract: a case-based imaging review with pathological correlation. Insights Imaging 2015;6:273.
7. Chun YK. Neuroendocrine tumors of the female reproductive tract: a literature review. J Pathol Transl Med 2015; doi: 10.4132/ jptm.2015.09.20.

8. Singh S, Asa SL, Dey C, Kennecke H, Laidley D, Law C, Asmis T, Chan D, Ezzat S, Goodwin R, Mete O, Pasieka J, Rivera J, Wong R, Segelov E, Rayson D. Diagnosis and management of gastrointestinal neuroendocrine tumors: an evidence-based Canadian consensus. Cancer Treat Rev 2016;47:32-45

9. Eads JR. Poorly differentiated neuroendocrine tumors. Hematol Oncol Clin North Am 2016;30:151-62.

10. Satake T, Matsuyama M. Argyrophil cells in normal endometrial glands. Virchows Arch A Pathol Anat Histopathol 1987;410:449-54.

11. Shaco-Levy R, Manor E, Piura B, Ariel I. An unusual composite endometrial tumor combining papillary serous carcinoma and small cell carcinoma. Am J Surg Pathol 2004;28:1103-6.

12. Taraif SH, Deavers MT, Malpica A, Silva EG. The significance of neuroendocrine expression in undifferentiated carcinoma of the endometrium. Int J Gynecol Pathol 2009;28:142-7.

13. Tafe LJ, Garg K, Chew I, Tornos C, Soslow RA. Endometrial and ovarian carcinomas with undifferentiated components: clinically aggressive and frequently underrecognized neoplasms. Mod Pathol 2010;23:781-9.

14. Ieni A, Barresi V, Branca G, Giuffrè G, Rosa MA, Tuccari G Immunoexpression of lactoferrin in bone metastases and corresponding primary carcinomas. Oncol Lett 2013;5:1536-40.

15. Ieni A, Barresi V, Grosso M, Rosa MA, Tuccari G. Lactoferrin immuno-expression in human normal and neoplastic bone tissue. $J$ Bone Miner Metab 2009;27:364-71.

16. Cohen JG, Kapp DS, Shin JY, Urban R, Sherman AE, Chen LM, Osann $\mathrm{K}$, Chan JK. Small cell carcinoma of the cervix: treatment and survival outcomes of 188 patients. Am J Obstet Gynecol 2010;203:347.e1-6.

17. Pocrnich CE, Ramalingam P, Euscher ED, Malpica A. Neuroendocrine carcinoma of the endometrium: a clinicopathologic study of 25 cases. Am J Surg Pathol 2016;40:577-86.

18. Sahnane N, Furlan D, Monti M, Romualdi C, Vanoli A, Vicari E Solcia E, Capella C, Sessa F, La Rosa S. Microsatellite unstable gastrointestinal neuroendocrine carcinomas: a new clinicopathologic entity. Endocr Relat Cancer 2015;22:35-45.

19. Gardner GJ, Reidy-Lagunes D, Gehrig PA. Neuroendocrine tumors of the gynecologic tract: a Society of Gynecologic Oncology (SGO) clinical document. Gynecol Oncol 2011;122:190-8. 